\title{
Recent Advances in Multiple Myeloma
}

\author{
An Expert Interview with Shaji K Kumar
}

Mayo Clinic, Rochester, Minnesota, US

DOI: https://doi.org/10.17925/OHR.2017.13.01.13

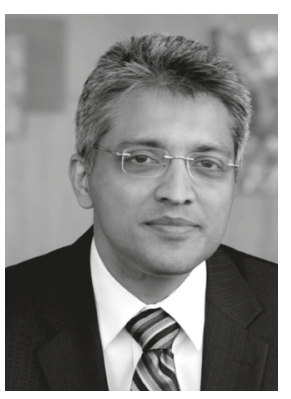

O

ncology \& Hematology Review (US) are delighted to introduce our new Editor-in-Chief, Shaji K Kumar, consultant in the Division of Hematology at Mayo Clinic, and Professor of Medicine in the College of Medicine, Mayo Clinic, Rochester, Minnesota, US. In a recent interview, Dr Kumar discussed the latest advances in multiple myeloma, one of his major research interests.

\section{Keywords}

Multiple myeloma, disease

progression, immunotherapy

Disclosure: Shaji K Kumar has nothing to disclose in relation to this article. This is an expert interview, and as such has not undergone the journal's standard peer review process.

Acknowledgments: Medical writing assistance was provided by Katrina Mountfort, Freelance Medical Writer, supported by Touch Medical Media.

Authorship: All named authors meet the International Committee of Medical Journal Editors (ICMJE) criteria for authorship of this manuscript, take responsibility for the integrity of the work as a whole, and have given final approval to the version to be published. Open Access: This article is published under the Creative Commons Attribution Noncommercial License, which permits any noncommercial use, distribution, adaptation, and reproduction provided the original author(s) and source are given appropriate credit.

Received: February 27, 2017

Published Online: April 14, 2017

Citation: Oncology \& Hematology Review, 2017;13(1):13-4

Corresponding Author: Shaji K Kumar, MD, Professor of Medicine, Division of Hematology, Mayo Clinic, 200 First Street SW, Rochester, MN 55905, US. E: kumar.shaji@mayo.edu
Oncology and Hematology Review (US) would like to welcome our new Editor-in-Chief, Shaji K Kumar, MD. Dr Kumar is a consultant in the Division of Hematology at Mayo Clinic, and Professor of Medicine in the College of Medicine, Mayo Clinic, Rochester, Minnesota, US. He is currently the chair of the NCCN panel on multiple myeloma and is the co-chair of the $\mathrm{NCl}$ multiple myeloma steering committee. A significant component of Dr Kumar's research efforts are directed toward studying novel treatment approaches in myeloma through well-designed clinical trials. He is principal investigator on multiple phase I, II, and III trials for plasma cell malignancies, which include a combination of Mayo investigator-initiated trials, industry-sponsored trials, and cooperative group trials. From a translational research standpoint, his laboratory has focused on development of new agents for plasma cell malignancies, with a particular focus on the tumor microenvironment. Dr Kumar receives ongoing support for research from the National Institutes of Health and the National Cancer Institute. He is a member of several professional societies, including the American Society of Hematology, American Society of Clinical Oncology, American Association for Clinical Research, American Society for Blood and Marrow Transplantation, American Medical Association, Association of Physicians of India, and the European Hematology Association. His work has appeared in peer reviewed journals such as Lancet Oncology, Journal of Clinical Oncology, Blood Cancer Journal, Leukemia, American Journal of Hematology, Mayo Clinic Proceedings, Blood, and European Journal of Hematology. He received his medical degree from the All India Institute of Medical Sciences, New Delhi, India. He completed an internship there, followed by a clinical residency in internal medicine and a senior residency in medical oncology. Dr Kumar subsequently completed a residency in internal medicine and a fellowship in hematology/oncology at Mayo Graduate School of Medicine. He then spent time as a research associate and Mayo Foundation Scholar in multiple myeloma at Dana-Farber Cancer Institute-Harvard Cancer Center. 


\section{Q: What are the most promising new therapies in clinical development for multiple myeloma?}

We have been fortunate to have a steady pipeline of novel therapies being developed in myeloma, which has resulted in the improved survival we see in the disease today. Among the more recent therapies being evaluated in clinical trials, there are several exciting classes that are showing early promise. Immunotherapy approaches have now come of age in myeloma. We had the first monoclonal antibodies approved for use in myeloma in the past year, daratumumab and elotozumab. Recent studies have shown that checkpoint inhibitors may have a role in myeloma, with two separate studies of pembrolizumab with pomalidomide or lenalidomide showing efficacy in relapsed myeloma. Early data from chimeric antigen receptor (CAR) T-cell trials demonstrate striking responses, and several B-cell maturation antigen (BCMA) targeted constructs are currently in clinical trial. Venetoclax ${ }^{\mathrm{TM}}$ (AbbVie, Inc., Chicago, US), a B-cell Iymphoma 2 (BCL 2) inhibitor, has shown striking activity as a single agent in patients with $t(11 ; 14)$ and in all patients in combination with bortezomib. This has moved to phase III and raises the possibility of a targeted therapy in multiple myeloma (MM). Selinexor, an exportin 1 (XPO1) inhibitor, inhibits the transport of tumor suppressor proteins, and can result in clinical responses in $20 \%$ of patients who are refractory to other available classes of drugs.

\section{Q: What have been the latest findings on driver mutations linked to disease progression and resistance to current therapies?}

We starting to understand the genetic landscape of myeloma and the precursor conditions. The spectrum of genetic abnormalities, including structural and numeric chromosomal abnormalities, and genetic mutations, has been the focus of several large efforts in the recent past. This has led to an appreciation of the genetic heterogeneity seen in this disease, leading us to the conclusion that myeloma is not just one disease, but a collection of plasma cell malignancies driven by different set of abnormalities. However, a single driver of disease etiology and for progression has not been identified. Mutations that can lead to drug resistance and disease progression have been identified and this is starting to translate to development of targeted therapies in MM.

\section{Q: What is the role of minimal residual disease testing in the management of multiple myeloma?}

Minimal residual disease is an old concept in hematology, but relatively new to myeloma, where its achievement had been hampered by lack of very effective therapies until now. With the combinations in use in myeloma, we are able to achieve deep responses, well beyond the discriminatory ability of traditional measurements of monoclonal protein or morphological assessment of the bone marrow. Using multicolor flow or next generation sequencing, we are able to detect less than one in a million myeloma cells in the bone marrow, and together with imaging to detect minimal amounts of disease outside the marrow, minimal residual disease (MRD) nativity has been defined and added to the current consensus response criteria. Based on the current data, we believe that it can be a valid surrogate endpoint for clinical trials to predict survival outcomes. Whether it can be used to guide therapy for an individual patient remains unclear and is the subject of future clinical trials.

\section{Q: What are the latest developments in identifying molecular predictors of outcome and drug response in multiple myeloma?}

Understanding the biology of disease and mechanism-of-action of drugs will allow us to maximize the use of a particular drug in myeloma. A better understanding of the targets of the currently available class of drugs has enabled us to start developing novel approaches to reversing drug resistance. In the setting of IMiDS ${ }^{\circledR}$ (Celgene, New Jersey, US), identification of cereblon as a target of the drug has led to identification of several important downstream pathways. Development of Venetoclax highlights another opportunity for targeted therapy that can be selected based on the molecular characteristics of the tumor cells.

\section{Q: Could you highlight any other developments we may expect to hear about in 2017 ?}

Advances in the treatments will continue to headline the progress in myeloma, However, more sensitive techniques of disease measurement such as mass spec based assays for detecting very small amounts of monoclonal protein as well as blood based assays for detection of circulating tumor cells or DNA has the potential to identify more stringent MRD negative status. Increasingly there is a pivot towards early intervention in myeloma, and several trials are looking at the possibility of treating smoldering myeloma with the goal of potentially curing the disease. 